\title{
Potencial do uso de quatro espécies de bambu para a produção de carvão vegetal para uso doméstico
}

\author{
Potential of the use of four bamboo species for the production of charcoal for \\ home use
}

\author{
Martha Andreia Brand', Angela Zakostelsky Neves GaaI", \\ Ailton Leonel Balduino Junior ${ }^{\mathrm{III}}$, Alexsandro Bayestorff da Cunha ${ }^{\mathrm{I}}$, \\ Polliana D'Angelo Rios ${ }^{\mathrm{I}}$
}

\begin{abstract}
Resumo
A utilização de colmos de bambu pode ser uma importante alternativa de ampliação das fontes renováveis para a produção de bioenergia, inclusive para a produção de carvão. Assim, o objetivo deste trabalho foi avaliar o potencial de quatro espécies de bambu para a produção de carvão vegetal para uso doméstico, como forma de promoção do uso múltiplo desta matéria-prima e para geração de renda adicional para os agricultores familiares de Santa Catarina. Foram analisados cinco indivíduos das espécies Bambusa vulgaris, coletada em Florianópolis, e Phyllostachys bambusoides; Phyllostachys edulis e Phyllostachys nigra coletadas no município de Frei Rogério, Santa Catarina. As análises da massa específica básica dos colmos in natura, as propriedades físicas, rendimento da carbonização e propriedades energéticas do carvão foram realizadas nas porções basal, mediana e topo dos colmos de bambu de cada espécie. A qualidade do carvão vegetal variou entre as espécies de bambu, tendo como único ponto negativo o alto teor de cinzas para uso doméstico do carvão vegetal. Houve alta correlação positiva entre a densidade básica dos colmos e a densidade relativa aparente do carvão vegetal. A espécie Bambusa vulgaris apresentou o maior rendimento no processo produtivo, porém gerou carvão mais leve e com pior desempenho energético. Entre as espécies avaliadas, Phyllostachys nigra foi a espécie que produziu o carvão vegetal com a melhor qualidade energética e com boas propriedades físicas e rendimento gravimétrico. A qualidade do carvão vegetal variou em relação à altura do colmo. O topo dos colmos, que é o resíduo que pode ser destinado para a produção de carvão em plantios comerciais de bambu para múltiplos usos, apresentou maior rendimento no processo produtivo e carvão com maior densidade. Porém a qualidade energética do carvão foi inferior em relação a base e posição mediana dos colmos.
\end{abstract}

Palavras-chaves: Análise imediata; Poder calorífico superior; Densidade aparente; Rendimento gravimétrico

\footnotetext{
Engenheiro Florestal, Dr., Universidade do Estado de Santa Catarina, Av. Luiz de Camões, 2090, CEP 88520- 000, Lages (SC), Brasil. martha.brand@ udesc.br (ORCID: 0000-0001-5438-2081) / alexsandro.cunha@udesc.br (ORCID: 0000-0001-5554-5276) / polliana.rios@udesc.br (ORCID: 0000-00023700-7084)

II Engenheira Industrial Madeireira, Pesquisadora Autônoma, Av. Luiz de Camões, 2090, CEP 88520- 000, Lages (SC), Brasil. espacoa.design@gmail. com (ORCID: 0000-0002-2377-9622)

III Engenheiro Industrial Madeireiro, MSc., Curso de Mestrado em Engenharia Florestal, Universidade do Estado de Santa Catarina, Av. Luiz de Camões, 2090, CEP 88520- 000, Lages (SC), Brasil. balduino.ailton@gmail.com (ORCID: 0000-0002-5621-154X)
} 


\begin{abstract}
The use of bamboo stems can be an important alternative for expanding renewable sources for bioenergy production, including for charcoal production. Therefore, the aim of this research was to evaluate the potential of four bamboo species for the production of charcoal for home use, as a way to promote the multiple use of this raw material and to generate additional income for the family farmers of Santa Catarina state. Five individuals of the species Bambusa vulgaris, collected in Florianópolis, and Phyllostachys bambusoides; Phyllostachys edulis; Phyllostachys nigra were collected in the municipality of Frei Rogério, Santa Catarina. The analyses of the basic density of the in natura stems, the physical properties, gravimetric yield and energetic properties of the charcoal were carried out in the basal, median and top portions of the bamboo stems of each species. The quality of the charcoal varied among the bamboo species, having as the only negative point the high ash content for home use charcoal. There was a high positive correlation between the basic density of the stem and the apparent relative density of charcoal. The species $B$. vulgaris presented the highest yield in the productive process, but it generated lighter charcoal and with lower energy performance. Among the evaluated species, Phyllostachys nigra was the species that produced the charcoal with the best energetic quality and with good physical properties and gravimetric yield. The quality of the charcoal varies with respect to the height of the stem. The top of the stems, which is the residue that can be destined for the production of charcoal in commercial plantations of bamboo for multiple uses, presented greater yield in the productive process and charcoal with greater density. However, the energetic quality of the charcoal was inferior in relation to the base and median position of the stems.
\end{abstract}

Keywords: Proximate analysis; Gross calorific value; Bulk density; Gravimetric yield

\title{
Introdução
}

Os bambus são gramíneas perenes de tronco lenhoso que ocorrem naturalmente na maioria das regiões tropicais do mundo. Existem mais de 1200 espécies de bambus, capazes de ocupar um grande número de habitats diferentes (GUERRA et al., 2016), com rendimentos muito elevados, em torno de 40 toneladas por hectare/ano (INTERNATIONAL NETWORK FOR BAMBOO AND RATTAN, 2014).

As espécies de bambu apresentam características adequadas como combustível para processos de geração de energia térmica, com potencial de utilização em diversos setores industriais, com qualidade semelhante ou superior a dos cavacos de eucalipto, matéria-prima comumente usada em processos industriais (MARAFON; AMARAL; LEMOS, 2019). No entanto, deve-se atentar para os seus altos teores de cinzas, pois estas podem vir a se fundir nas paredes das caldeiras, causando isolamento do sistema de aquecimento (VALE; MOREIRA; MARTINS, 2017). Além disso, essa biomassa se constitui não de resíduos da indústria agrícola, mas de culturas produtoras de energia, sendo promissora para utilização em biorrefinarias, no Brasil (RAMBO; SCHMIDT; ERREIRA, 2015).

$\mathrm{O}$ bambu tem um grande potencial como recurso de bioenergia devido a sua alta produtividade e rápido crescimento (LIU; FEI; JIANG, 2014). Podendo ser colhido em menos de quatro anos, a colheita pode ser continua, tornando-se uma fonte de energia confiável (INTERNATIONAL NETWORK FOR BAMBOO AND RATTAN, 2014, LIU; FEI; JIANG, 2014). Além disso, o uso do bambu na agricultura de base familiar vem sendo incentivado desde a promulgação, em 2011, da Lei no 12.484, que dispõe sobre a Política Nacional de Incentivo ao Manejo Sustentável e ao Cultivo do Bambu (PNMCB). Essa lei tem como objetivo principal o desenvolvimento da cultura do bambu no Brasil por meio de práticas governamentais e da atuação de empreendedores privados. Dentre outras ações, o governo federal pretende trabalhar na implementação do incentivo à pesquisa e ao desenvolvimento tecnológico voltados para o manejo sustentado, o cultivo, os serviços ambientais e as aplicações dos produtos e subprodutos do bambu; incentivar o cultivo e a utilização do bambu pela agricultura familiar; estimular o comércio interno e externo de bambu e de seus subprodutos (BRASIL, 2011).

A diversidade de espécies de bambu e suas características morfológicas justificam a sua utilização, a qual é histórica na sociedade (CAMPOS et al., 2016). No Brasil, as espécies exóticas mais importantes economicamente são dos gêneros Bambusa e Phyllostachys.

A espécie Bambusa vulgaris Schrad. ex J.C.Wendl. foi introduzida pelos colonizadores portugueses. A migração japonesa também exerceu forte influência na introdução dessa cultura, principalmente das 
espécies do tipo alastrante, como é o caso do bambu "mossô" - Phyllostachys edulis (Carrière) J. Houz -, na primeira metade do Século XX (TOMBOLATO; GRECO; PINTO, 2012). A espécie Phyllostachys edulis (Carrière) J. Houz é utilizada no Brasil em projetos estruturais, alimentação (brotos comestíveis) e na movelaria na forma de bambu laminado colado. Já a espécie Phyllostachys bambusoides Siebold \& Zucc. foi provavelmente trazida para o Brasil também pelos imigrantes japoneses na primeira metade do Século XX. Por apresentar elevados valores de resistência mecânica, é utilizado para fins estruturais e mais recentemente aproveitado para a produção de móveis em bambu laminado colado (TOMBOLATO; GRECO; PINTO, 2012). Já o Phyllostachys nigra Muchisasa, tem utilização mais notória na ornamentação e paisagismo (PEREIRA NETO et al., 2009).

Em Santa Catarina, a espécie Bambusa vulgaris é mais comum na região litorânea do estado, enquanto as espécies Phyllostachys edulis, Phyllostachys bambusoides e Phyllostachys nigra estão sendo cultivadas no município de Frei Rogério, na região serrana do Estado em uma representativa comunidade japonesa de Santa Catarina. As espécies do gênero Phyllostachys estão se mostrando resistentes a geadas e já são comercializadas pelos agricultores tanto para fins alimentícios, dentro da própria comunidade nipônica, como para a produção de palitos de churrasco e de fósforo em indústrias deste setor da região. Principalmente a porção superior dos colmos (topo) fica no campo, após a colheita dos bambus com três anos de idade. Esse resíduo constitui um problema para a renovação dos bambusais, além de ser uma matéria-prima desperdiçada. Essa demanda de uso dos resíduos do cultivo do bambu pelos produtores motivou esta pesquisa, que objetivou avaliar o potencial de quatro espécies de bambu para a produção de carvão vegetal para uso doméstico, que seria mais uma forma de aproveitamento dessa biomassa e geração de renda adicional para os agricultores familiares de Santa Catarina, em diferentes regiões do estado.

\section{Material e métodos}

Foram analisados cinco indivíduos (colmos) de cada uma das espécies estudadas, com três anos de idade. A espécie Bambusa vulgaris foi coletada na Fazenda experimental da Ressacada, pertencente à Universidade Federal de Santa Catarina, em Florianópolis (27²4103.7”S 483233.8”W). As espécies Phyllostachys bambusoides, Phyllostachys edulis e Phyllostachys nigra foram coletadas em uma propriedade da comunidade japonesa da cidade de Frei Rogério, no estado de Santa Catarina (27¹3'19.5”S5044'13.4”W). A caracterização morfométrica de cada indivíduo é apresentada na Tabela 1, sendo que foram coletadas seções de 1,0 m de comprimento na base, meio e topo de todos os colmos.

Tabela 1 - Dados dendrométricos médios dos colmos das espécies Bambusa vulgaris, Phyllostachys bambusoides, Phyllostachys edulis e Phyllostachys nigra

Table 1 - Dendrometric data of culms of the Bambusa vulgaris, Phyllostachys bambusoides, Phyllostachys edulis and Phyllostachys nigra bamboo species

\begin{tabular}{lcccc}
\hline Espécie & $\begin{array}{c}\text { Bambusa } \\
\text { vulgaris }\end{array}$ & $\begin{array}{c}\text { Phyllostachys } \\
\text { bambusoides }\end{array}$ & $\begin{array}{c}\text { Phyllostachys } \\
\text { edulis }\end{array}$ & $\begin{array}{c}\text { Phyllostachys } \\
\text { nigra }\end{array}$ \\
\hline DAP $(\mathbf{c m})$ & 9,66 & 8,00 & 7,96 & 7,67 \\
$\begin{array}{l}\text { Altura total } \\
(\mathbf{m})\end{array}$ & 15,10 & 14,30 & 15,60 & 15,75 \\
\hline
\end{tabular}

Fonte: Autores (2019)

Em que: DAP = Diâmetro à altura do peito

As análises físicas e energéticas nas espécies foram realizadas nos colmos in natura e no carvão produzido em laboratório. Por espécie e de cada colmo foram extraídos 10 corpos de prova 
de aproximadamente $2 \mathrm{~cm}$ de largura x $4 \mathrm{~cm}$ de comprimento e espessura variável, em cada porção do colmo (base, meio e topo), totalizando 30 corpos de prova por colmo e 150 por espécie, para a determinação da massa específica básica (MEB) por meio do método de máximo teor de umidade, segundo a norma ABNT NBR 11941 (2003a).

Os corpos de prova, nos quais foi feita a determinação da densidade básica, foram utilizados para a produção do carvão vegetal. A carbonização dos corpos de prova, logo após a determinação da densidade básica não teve a influência do teor de umidade do bambu no processo produtivo, que poderia afetar o rendimento gravimétrico e a composição química imediata de forma positiva.

A carbonização foi conduzida em um forno tipo mufla. Antes da carbonização, cada corpo de prova foi envolvido em papel alumínio, para impedir a entrada de ar. O tempo total da carbonização foi de 6 horas e 30 minutos, com uma temperatura final de $450^{\circ} \mathrm{C}$ nos últimos 30 minutos, e taxa de aquecimento variável ao longo da pirólise (Tabela 2).

Tabela 2 - Rampa de Carbonização utilizada para a produção de carvão vegetal de bambu

Table 2 - Ramp Charring used for the charcoal production of bamboo charcoal

\begin{tabular}{lccccccc}
\hline \multicolumn{1}{c}{ Tempo $($ horas$)$} & Inicio & $\mathbf{0 0 : 2 0}$ & $\mathbf{0 1 : 2 7}$ & $\mathbf{0 3 : 0 4}$ & $\mathbf{0 4 : 4 8}$ & $\mathbf{0 6 : 0 2}$ & $\mathbf{0 6 : 3 2}$ \\
\hline Temperatura $\left({ }^{\circ} \mathrm{C}\right)$ & 25 & 150 & 200 & 250 & 350 & 450 & 450 \\
Taxa de aquecimento $\left({ }^{\circ} \mathrm{C} / \mathbf{m i n}\right)$ & - & 7,5 & 2,3 & 1,36 & 1,22 & 1,24 & - \\
\hline
\end{tabular}

Fonte: Autores (2019)

Finalizada a carbonização, e após o resfriamento dos corpos de prova, foram obtidas as medidas lineares de comprimento, largura e espessura, e o produto dessas medidas resultou no volume do carvão $(\mathrm{Vc})$. A massa dos corpos-de-prova (peso seco do carvão) foi obtida pela pesagem em balança de precisão $(0,001 \mathrm{~g})(\mathrm{Mc})$. A relação entre a massa e o volume dos corpos de prova resultou na densidade aparente do carvão (DA) (Equação 1)

$$
\mathrm{DA}=\mathrm{Mc} / \mathrm{Vc}
$$

Onde: DA = densidade aparente do carvão $\left(\mathrm{g} \cdot \mathrm{cm}^{-3}\right) ; \mathrm{Mc}=$ massa do carvão vegetal $(\mathrm{g}) ; \mathrm{Vc}=$ volume do carvão vegetal $\left(\mathrm{cm}^{3}\right)$.

O rendimento gravimétrico da carbonização foi determinado pela razão entre o peso seco do carvão e o peso absolutamente seco dos corpos de prova antes da carbonização, obtido durante o processo de determinação da densidade básica, após secagem das amostras em estufa a $103 \pm 2{ }^{\circ} \mathrm{C}$. Com partículas de carvão moído realizou-se a determinação do teor de umidade, de acordo com a norma ABNT NBR 14929 (2003b); o poder calorífico superior, segundo a norma DIN 51900 (2000) e a análise imediata de acordo com a norma ASTM 1762 (2013).

Os valores médios das propriedades analisadas de cada espécie e nas diferentes posições dos colmos foram avaliados estatisticamente por meio do Teste $\mathrm{F}$, e se verificada variação nos tratamentos, foi aplicado o Teste de Médias de Scott-knott a 95\% de probabilidade. Além disso, foi aplicada análise de regressão e o coeficiente de correlação de Pearson para estabelecer as prováveis correlações entre as variáveis.

\section{Resultados e discussões}

Os resultados da massa específica básica dos colmos in natura, do rendimento gravimétrico e da densidade relativa aparente do carvão vegetal do bambu podem ser visualizados na Tabela 3. 
Quanto à densidade básica, como base na classificação do Laboratório de Produtos Florestais Brasileiro (2017), as espécies Bambusa vulgaris e Phyllostachys bambusoides podem ser classificadas como de densidade média por apresentarem valores entre 0,5 a $0,72{\mathrm{~g} . \mathrm{cm}^{-3}}^{-3}$, enquanto as espécies Phyllostachys edulis e Phyllostachys nigra podem ser classificadas com de alta densidade, por possuírem valores superiores a $0,72 \mathrm{~g} . \mathrm{cm}^{-3}$.

A análise das quatro espécies demonstrou que existiu variação significativa para a densidade básica média entre todas as espécies, com maiores valores para as espécies do gênero Phyllostachys.

Tabela 3 - Propriedades físicas dos colmos e parâmetros do processo de carbonização das espécies de bambu analisadas

Table 3 - Physical properties of the stem and the parameters of the carbonization process of the analyzed bamboo species

\begin{tabular}{|c|c|c|c|c|c|c|}
\hline \multicolumn{7}{|c|}{ Massa específica básica dos colmos (g.cm³) } \\
\hline Posição & $\begin{array}{c}\text { Bambusa } \\
\text { vulgaris }\end{array}$ & $\begin{array}{l}\text { Phyllostachys } \\
\text { bambusoides }\end{array}$ & $\begin{array}{c}\text { Phyllostachys } \\
\text { edulis }\end{array}$ & $\begin{array}{c}\text { Phyllostachys } \\
\text { nigra }\end{array}$ & Média & CV (\%) \\
\hline Base & В 0,539 c & В $0,701 \mathrm{~b}$ & B $0,822 \mathrm{a}$ & $\mathrm{C} 0,705 \mathrm{~b}$ & C 0,692 & 9,50 \\
\hline Meio & A $0,641 \mathrm{~d}$ & B 0,704 c & A 0,847 a & B $0,772 \mathrm{~b}$ & В 0,741 & 9,69 \\
\hline Topo & A $0,689 \mathrm{~d}$ & A 0,753 c & A 0,854 a & A $0,804 \mathrm{~b}$ & A 0,775 & 11,56 \\
\hline Média & $0,623 \mathrm{~d}$ & $0,719 \mathrm{c}$ & $0,841 \mathrm{a}$ & $0,761 \mathrm{~b}$ & 0,736 & \multirow[t]{2}{*}{11,65} \\
\hline CV (\%) & 21,93 & 5,55 & 4,30 & 5,52 & 15,11 & \\
\hline \multicolumn{7}{|c|}{ Densidade relativa aparente do carvão $\left({\left.\mathrm{g} . \mathrm{cm}^{-3}\right)}^{-}\right.$} \\
\hline Posição & $\begin{array}{c}\text { Bambusa } \\
\text { vulgaris }\end{array}$ & $\begin{array}{c}\text { Phyllostachys } \\
\text { bambusoides }\end{array}$ & $\begin{array}{c}\text { Phyllostachys } \\
\text { edulis }\end{array}$ & $\begin{array}{c}\text { Phyllostachys } \\
\text { nigra }\end{array}$ & Média & CV (\%) \\
\hline Base & C $0,323 \mathrm{~d}$ & A 0,354 c & C $0,513 \mathrm{a}$ & $\mathrm{C} 0,419 \mathrm{~b}$ & C 0,402 & 16,34 \\
\hline Meio & B 0,363 c & A 0,373 c & B 0,598 a & B $0,451 \mathrm{~b}$ & B 0,446 & 13,91 \\
\hline Topo & A 0,431 c & A $0,375 \mathrm{~d}$ & A 0,625 a & A $0,547 \mathrm{~b}$ & A 0,494 & 14,70 \\
\hline Média & $0,373 \mathrm{c}$ & $0,367 \mathrm{c}$ & $0,579 \mathrm{a}$ & $0,472 \mathrm{~b}$ & 0,448 & \multirow[t]{2}{*}{17,68} \\
\hline CV (\%) & 22,27 & 15,24 & 11,25 & 12,86 & 24,81 & \\
\hline \multicolumn{7}{|c|}{ Rendimento gravimétrico (\%) } \\
\hline Posição & $\begin{array}{c}\text { Bambusa } \\
\text { vulgaris }\end{array}$ & $\begin{array}{l}\text { Phyllostachys } \\
\text { bambusoides }\end{array}$ & $\begin{array}{c}\text { Phyllostachys } \\
\text { edulis }\end{array}$ & $\begin{array}{c}\text { Phyllostachys } \\
\text { nigra }\end{array}$ & Média & $\mathrm{CV}(\%)$ \\
\hline Base & A 37,14 a & A 33.29 c & B 32,78 c & A $34,89 \mathrm{~b}$ & В 34,52 & 6,03 \\
\hline Meio & B 35,49 a & A 33,17 c & B 33,29 c & A $34,63 \mathrm{~b}$ & B 34,15 & 4,79 \\
\hline Topo & A $36,57 \mathrm{a}$ & A $33,53 \mathrm{~d}$ & A $34,40 \mathrm{c}$ & A $35,19 \mathrm{~b}$ & A 34,92 & 6,20 \\
\hline Média & 36,40 a & $33,33 \mathrm{c}$ & $33,49 \mathrm{c}$ & $34,90 \mathrm{~b}$ & 34,53 & \multirow[t]{2}{*}{5,86} \\
\hline CV (\%) & 6,15 & 3,66 & 7,74 & 4,42 & 6,81 & \\
\hline
\end{tabular}

Fonte: Autores (2019)

Em que: $C V$ = coeficiente de variação. Médias precedidas de letras MAIÚSCULAS iguais, na COLUNA, indicam que não houve variação significativa entre as posições do colmo (base, meio e topo). Médias seguidas de letras MINÚSCULAS iguais, na LINHA, indicam que não houve variação significativa entre as espécies. 
Em relação à altura do colmo, também houve variação entre as espécies, para cada posição (base, meio e topo). Na base, as espécies Phyllostachys nigra e Phyllostachys bambusoides foram semelhantes entre si. Para o meio e topo dos colmos, todas as espécies foram diferentes entre si. Os maiores valores foram observados nos colmos de Phyllostachys edulis em todas as posições, e no valor médio da espécie. Os menores valores, em todas as posições e no valor médio, foram para Bambusa vulgaris.

Em cada espécie, a densidade básica diferiu na altura do colmo, sendo maior no topo e menor na base, podendo a porção mediana ser semelhante à base (Phyllostachys bambusoides), semelhante ao topo (Phyllostachys edulis e Bambusa vulgaris) ou diferente das demais posições (Phyllostachys nigra). O bambu é uma monocotiledônea, apresentando somente o crescimento primário, sem formação de xilema secundário, tendo, portanto, o comportamento inverso ao de espécies madeiráveis. Esse comportamento também já foi observado por Berndsen et al. (2010).

Para a geração de energia esse comportamento pode ser positivo, tornando as espécies de bambu potenciais materiais. Isso porque, na região de plantio das espécies estudadas, a base e o meio dos colmos são comercializados para a fabricação de palitos de fósforo, palitos de churrasco e para fabricação de painéis, ficando os topos do colmo no campo sem aproveitamento. Além disso, os resíduos deixados em campo dificultam as atividades de manejo dos plantios, que sofrem intervenção a cada três anos, que é o ciclo de corte, visto que os colmos com essa idade são considerados adultos e maduros.

Para Bambusa vulgaris, Melo et al. (2015) obtiveram o valor médio de densidade básica de 0,630 g. $\mathrm{cm}^{-3}$, para colmos com aproximadamente quatro anos de idade, enquanto Marafon, Amaral e Lemos (2019) obtiveram valores médios de $0,638 \mathrm{~g} . \mathrm{cm}^{-3}$. Os valores de literatura foram próximos dos observados neste trabalho. Para Phyllostachys pubescens (sinonímia do Phyllostachys edulis), Berndsen et al. (2010) encontraram valor médio de densidade básica de $0,67 \mathrm{~g}_{\mathrm{cm}} \mathrm{cm}^{-3}$, para colmos com 3 anos de idade e valores de 0,$63 ; 0,69$ e $0,71 \mathrm{~g} \cdot \mathrm{cm}^{-3}$ para as posições base, meio e topo dos colmos, respectivamente, que foram inferiores aos observados neste trabalho.

Neste estudo houve correlação positiva entre a densidade básica dos colmos e a densidade aparente do carvão, como também mencionado por Santos et al. (2011). O Phyllostachys edulis, que teve a maior densidade básica também teve a maior média de densidade aparente do carvão vegetal, seguido do Phyllostachys nigra, sendo o Phyllostachys bambusoides e o Bambusa vulgaris estatisticamente iguais para a densidade aparente do carvão vegetal. Para a análise da relação entre as variáveis foram analisadas 600 amostras englobando as quatro espécies e houve correlação positiva entre as variáveis como pode ser visualizado na Figura 1, tendo-se um $\mathrm{R}^{2}$ de 0,67 com ajuste polinomial de segunda ordem.

\section{Figura 1 - Relação entre a densidade aparente do carvão vegetal e a densidade básica dos colmos de bambu de diferentes espécies}

Figure 1 - Relationship between the apparent density of the charcoal and the basic density of bamboo culms of different species

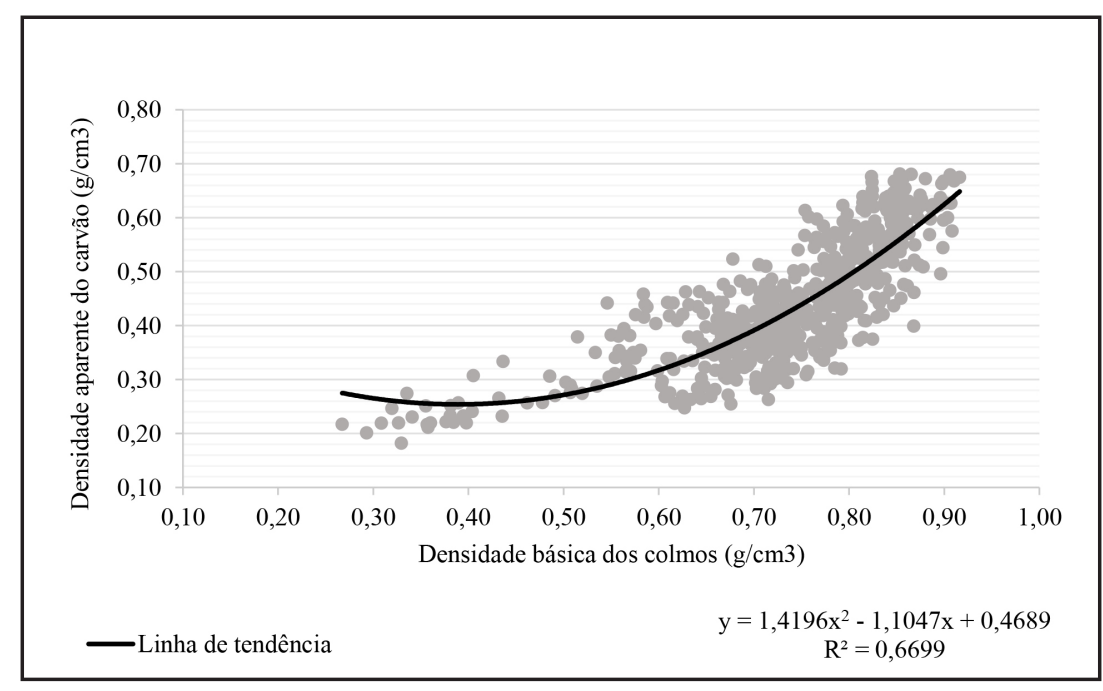

Fonte: Autores (2019) 
Para a densidade aparente do carvão em cada posição do colmo, as variações observadas entre as espécies seguiram a mesma tendência dos valores médios, sendo as espécies Bambusa vulgaris e Phyllostachys bambusoides iguais entre si, na posição meio e diferentes nas posições meio e topo. As espécies Phyllostachys edulis e Phyllostachys nigra foram diferentes entre si e com as demais espécies em todas as posições do colmo.

A variação em relação a posição do colmo, considerando de forma conjunta todas as espécies, seguiu a mesma tendência da densidade básica, ou seja, a densidade é maior no topo, decrescendo gradativamente para a base. De forma geral, a densidade aparente do carvão vegetal de bambu foi alta, sendo maior que os valores apresentados por Santos et al. (2011) que trabalharam com clones de espécies de Eucalyptus com 7 anos de idade e obtiveram valores entre 0,266 e $0,345 \mathrm{~g} \cdot \mathrm{cm}^{-3}$.

O rendimento gravimétrico do carvão das quatro espécies foi alto quando comparado aos valores observados por Santos et al. (2011) que variaram entre $28,27 \%$ a $30,21 \%$, e os valores registrados por Neves et al. (2011), entre 30,26 a 32,47\%, também para Eucalyptus. Santana et al. (2019) analisando a qualidade do carvão de Bambusa vulgaris encontraram valores de 35,83\% de rendimento gravimétrico, inferior ao observado neste trabalho. A espécie Bambusa vulgaris teve o maior rendimento, sendo a espécie com a menor densidade básica e juntamente com o Phyllostachys bambusoides teve as menores densidades aparentes do carvão. As espécies Phyllostachys bambusoides e Phyllostachys edulis foram semelhantes entre si com os menores valores de rendimento gravimétrico. A correlação entre o rendimento gravimétrico e densidade básica do bambu foi baixa $\left(\mathrm{R}^{2}=0,20\right)$, como também entre o rendimento gravimétrico e a densidade aparente do carvão $\left(\mathrm{R}^{2}=0,02\right)$.

Para o rendimento gravimétrico do carvão em cada posição do colmo, as espécies Phyllostachys bambusoides e Phyllostachys nigra não tiveram variação ao longo da altura dos colmos. Para os valores médios, considerando as espécies de forma conjunta, o rendimento foi maior no topo, sendo a base e meio semelhantes entre si.

A Tabela 4 mostra o teor de umidade e o poder calorífico superior do carvão produzido com as quatro espécies estudadas.

Tabela 4 - Teor de umidade e poder calorífico superior do carvão vegetal produzido com diferentes espécies de bambu

Table 4 - Moisture content and gross calorific value of charcoal produced with different species of bamboo

\begin{tabular}{l|c|c|c|c|c|c}
\hline \multicolumn{1}{c}{ Teor de umidade do carvão (\%) } \\
\hline Posição & $\begin{array}{c}\text { Bambusa } \\
\text { vulgaris }\end{array}$ & $\begin{array}{c}\text { Phyllostachys } \\
\text { bambusoides }\end{array}$ & $\begin{array}{c}\text { Phyllostachys } \\
\text { edulis }\end{array}$ & $\begin{array}{c}\text { Phyllostachys } \\
\text { nigra }\end{array}$ & Média & CV (\%) \\
\hline Base & A 4,62 b & A 3,71 b & A 10,18 a & B 4,23 b & A 5,68 & 18,51 \\
Meio & A 4,78 b & A 4,60 b & A 9,95 a & B 4,26 b & A 5,90 & 22,29 \\
Topo & A 4,62 b & A 5,22 b & A 9,13 a & A 4,73 b & A 5,92 & 20,96 \\
\hline Média & 4,67 b & 4,51 b & 9,75 a & 4,40 b & 5,83 & 21,38 \\
\hline CV (\%) & 20,97 & 38,06 & 13,26 & 11,44 & 44,53 &
\end{tabular}


Tabela 4 - Conclusão ...

Table 4 - Conclusion ...

\begin{tabular}{l|c|c|c|c|c|c}
\hline \multicolumn{1}{c}{ Poder calorífico superior (Kcal.kg-1) } \\
\hline Posição & $\begin{array}{c}\text { Bambusa } \\
\text { vulgaris }\end{array}$ & $\begin{array}{c}\text { Phyllostachys } \\
\text { bambusoides }\end{array}$ & $\begin{array}{c}\text { Phyllostachys } \\
\text { edulis }\end{array}$ & $\begin{array}{c}\text { Phyllostachys } \\
\text { nigra }\end{array}$ & Média & CV (\%) \\
\hline Base & A 7621 a & A 7675 a & A 7416 b & B 7563 a & A 7569 & 2,90 \\
Meio & A 7470 b & A 7631 a & A 7453 b & A 7721 a & A 7569 & 2,77 \\
Topo & B 7200 b & A 7457 a & A 7404 a & B 7490 a & B 7388 & 4,18 \\
\hline Média & $7431 \mathrm{~b}$ & $7588 \mathrm{a}$ & $7424 \mathrm{~b}$ & $7591 \mathrm{a}$ & 7508 & 3,55 \\
\hline CV $(\%)$ & 3,05 & 3,94 & 3,66 & 2,50 & 3,58 & \\
\hline
\end{tabular}

Fonte: Autores (2019)

Em que: $\mathrm{CV}$ = coeficiente de variação. Médias precedidas de letras MAIÚSCULAS iguais, na COLUNA, indicam que não houve variação significativa entre as posições do colmo (base, meio e topo). Médias seguidas de letras MINÚSCULAS iguais, na LINHA, indicam que não houve variação significativa entre as espécies.

O teor de umidade do carvão produzido com as espécies de bambu foi inferior a $5 \%$ de umidade para as espécies Bambusa vulgaris, Phyllostachys bambusoides e Phyllostachys nigra, que foram semelhantes entre si, atendendo aos critérios de qualidade estabelecidos pelo Selo Premium, promulgado pela Resolução ${ }^{\circ} 10$ SAA, de 11 de julho de 2003, no Estado de São Paulo (2003), que determina que a umidade do carvão vegetal deve estar abaixo de $5 \%$ para uso doméstico. Em relação à posição do colmo, somente a espécie Phyllostachys nigra teve diferença de teor de umidade entre o topo, com maior valor, e as demais posições que foram semelhantes entre si.

O poder calorífico do carvão vegetal foi alto, havendo diferença entre as espécies analisadas, com semelhança estatística entre o Phyllostachys bambusoides e Phyllostachys nigra que foram diferentes do outro grupo de similaridade formado pelo Phyllostachys edulis e Bambusa vulgaris, que apresentaram menores valores de poder calorífico. Para as espécies Phyllostachys bambusoides e Phyllostachys edulis não houve diferença estatística entre as posições do colmo, enquanto para as espécies Phyllostachys nigra e Bambusa vulgaris o poder calorífico foi menor no topo.

De forma geral, pode ser observada a tendência de redução do poder calorífico da base para o topo dos colmos nas quatro espécies avaliadas. Segundo Oyedun, Gebreegziabher e Hui (2013) o carvão de bambu é superior a outras fontes de carvão em termos de valores caloríficos. Os resultados aqui obtidos foram comparados com os valores de literatura, cujas pesquisas aplicaram os mesmos parâmetros de carbonização (temperatura, taxa de aquecimento e tempo de carbonização) utilizados neste trabalho. Os valores do carvão de bambu foram similares aos valores obtidos por Neves et al. (2011), para carvão de Eucalyptus, que variaram entre 7625 a 7698

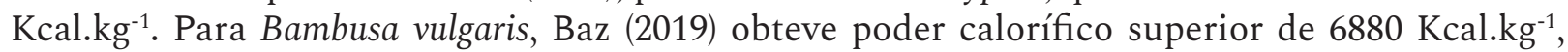
inferior ao da mesma espécie deste trabalho.

Considerando a composição química imediata do carvão vegetal (Tabela 5), o teor de voláteis médio foi alto, havendo diferença na qualidade do carvão entre as espécies. O Bambusa vulgaris foi semelhante estatisticamente ao Phyllostachys bambusoides, sendo as duas outras espécies avaliadas diferentes entre si e com o grupo anterior. Para carbono fixo, o valor médio foi baixo, e todas as espécies foram diferentes entre si. O teor de cinzas foi alto, com maior valor para Bambusa vulgaris, diferente das demais espécies, e os menores valores foram das espécies Phyllostachys bambusoides e Phyllostachys edulis, semelhantes entre si. Andrade et al. (2015) analisando a qualidade do carvão de Bambusa vulgaris encontraram valores de 64,11\% de carbono 
fixo, $28,55 \%$ de voláteis, $4,8 \%$ de cinzas, sendo o carvão de pior qualidade em comparação com o obtido neste trabalho, com exceção do teor de cinzas.

Para a composição química imediata do carvão vegetal, somente a espécie Phyllostachys nigra apresentou valores que atendem a Resolução n ${ }^{\circ} 10$ SAA (SÃO PAULO, 2003) que determina que o carvão para uso doméstico deve ter teor de carbono fixo superior a $75 \%$; teor de materiais voláteis e teor de cinzas inferiores a $23,5 \%$ e $1,5 \%$, respectivamente. Para o teor de cinzas, nenhuma das espécies produziu carvão com teor de cinzas abaixo de $1,5 \%$.

Tabela 5 -Composição química imediata do carvão produzido com diferentes espécies de bambu

Table 5 - Proximate composition of the charcoal produced with different species of bamboo

\begin{tabular}{|c|c|c|c|c|c|c|}
\hline \multicolumn{7}{|c|}{ Teor de voláteis $(\%)$} \\
\hline Posição & $\begin{array}{c}\text { Bambusa } \\
\text { vulgaris }\end{array}$ & $\begin{array}{l}\text { Phyllostachys } \\
\text { bambusoides }\end{array}$ & $\begin{array}{c}\text { Phyllostachys } \\
\text { edulis }\end{array}$ & $\begin{array}{c}\text { Phyllostachys } \\
\text { nigra }\end{array}$ & Média & CV (\%) \\
\hline Base & A 28,28 a & B 26,49 a & A $24,50 \mathrm{~b}$ & A $20,55 \mathrm{c}$ & B 24,95 & 10,55 \\
\hline Meio & B $26,01 \mathrm{a}$ & B $26,52 \mathrm{a}$ & A $24,74 \mathrm{~b}$ & A 20,86 c & B 24,53 & 8,28 \\
\hline Topo & A $28,37 \mathrm{a}$ & A 28,78 a & A $26,02 \mathrm{~b}$ & A $21,46 \mathrm{c}$ & A 26,16 & 8,25 \\
\hline Média & $27,55 \mathrm{a}$ & $27,26 \mathrm{a}$ & $25,09 \mathrm{~b}$ & $20,95 \mathrm{c}$ & 25,21 & 9,51 \\
\hline CV (\%) & 8,96 & 7,22 & 10,08 & 10,24 & 13,92 & \\
\hline \multicolumn{7}{|c|}{ Teor de carbono fixo (\%) } \\
\hline Posição & $\begin{array}{c}\text { Bambusa } \\
\text { vulgaris }\end{array}$ & $\begin{array}{c}\text { Phyllostachys } \\
\text { bambusoides }\end{array}$ & $\begin{array}{l}\text { Phyllostachys } \\
\text { edulis }\end{array}$ & $\begin{array}{c}\text { Phyllostachys } \\
\text { nigra }\end{array}$ & Média & CV (\%) \\
\hline Base & В 66,89 c & A $72,05 \mathrm{~b}$ & A $73,59 \mathrm{~b}$ & A $75,74 \mathrm{a}$ & A 72,07 & 3,82 \\
\hline Meio & A $69,22 \mathrm{c}$ & A $72,13 \mathrm{~b}$ & A $73,22 \mathrm{~b}$ & A $76,53 \mathrm{a}$ & A 72,77 & 2,95 \\
\hline Topo & B $65,86 \mathrm{~d}$ & B $69,08 \mathrm{c}$ & A 72,47 b & A $75,24 \mathrm{a}$ & B 70,66 & 3,40 \\
\hline Média & $67,32 \mathrm{~d}$ & $71,09 \mathrm{c}$ & $73,09 \mathrm{~b}$ & $75,84 \mathrm{a}$ & 71,83 & 3,65 \\
\hline CV (\%) & 4,39 & 2,57 & 3,76 & 2,75 & 5,54 & \\
\hline \multicolumn{7}{|c|}{ Teor de cinzas $(\%)$} \\
\hline Posição & $\begin{array}{c}\text { Bambusa } \\
\text { vulgaris }\end{array}$ & $\begin{array}{c}\text { Phyllostachys } \\
\text { bambusoides }\end{array}$ & $\begin{array}{c}\text { Phyllostachys } \\
\text { edulis }\end{array}$ & $\begin{array}{c}\text { Phyllostachys } \\
\text { nigra }\end{array}$ & Média & CV (\%) \\
\hline Base & B 4,83 a & B $1,46 \mathrm{c}$ & A $1,92 \mathrm{c}$ & A $3,71 \mathrm{~b}$ & A 2,98 & 24,67 \\
\hline Meio & B 4,77 a & B $1,35 \mathrm{~d}$ & A $2,04 \mathrm{c}$ & $\mathrm{C} 2,61 \mathrm{~b}$ & A 2,69 & 23,07 \\
\hline Topo & A $5,76 \mathrm{a}$ & A $2,14 \mathrm{c}$ & B $1,51 \mathrm{c}$ & B $3,30 \mathrm{~b}$ & A 3,18 & 30,68 \\
\hline Média & $5,12 \mathrm{a}$ & $1,65 \mathrm{c}$ & $1,82 \mathrm{c}$ & $3,21 \mathrm{~b}$ & 2,95 & 29,27 \\
\hline CV (\%) & 22,15 & 53,25 & 28,68 & 12,93 & 55,41 & \\
\hline
\end{tabular}

Fonte: Autores (2019)

Em que: $C V$ = coeficiente de variação. Médias precedidas de letras MAIÚSCULAS iguais, na COLUNA, indicam que não houve variação significativa entre as posições do colmo (base, meio e topo). Médias seguidas de letras MINÚSCULAS iguais, na LINHA, indicam que não houve variação significativa entre as espécies. 
Para as espécies Bambusa vulgaris e Phyllostachys bambusoides houve variação na composição química imediata ao longo do colmo, com maiores valores de teor de voláteis e menores de carbono fixo no topo em relação à posição mediana e basal. O teor de cinzas também foi maior no topo para ambas as espécies, sendo que o Phyllostachys bambusoides, produziu carvão com menor teor médio de cinzas. Já a espécie Bambusa vulgaris teve o maior teor médio de cinzas. As espécies Phyllostachys edulis e Phyllostachys nigra não tiveram variação no teor de voláteis e carbono fixo ao longo dos colmos.

Considerando, de forma geral, o uso doméstico para cocção de alimentos, o carvão de bambu teve como única propriedade desfavorável seu maior teor de cinzas em comparação com o carvão produzido a partir da madeira. Brand et al. (2015) analisaram 12 marcas de carvão vegetal derivado de espécies florestais comercializadas na mesma mesorregião de estudo, no Estado de Santa Catarina. Os autores constataram que o carvão vegetal de uso doméstico tinha valores de densidade relativa aparente média de $0,403 \mathrm{~g} \cdot \mathrm{cm}^{-3}(0,245$ a 0,488$)$; teor de umidade de $7,35 \%$ $(5,59$ a 8,21$)$; poder calorífico superior de $6449 \mathrm{kcal} / \mathrm{kg}$ (4301-7968); teor de voláteis de $32,85 \%$ $(26,15-39,47) ; 65,17 \%$ de carbono fixo $(61,66$ a 71,21$)$ e teor de cinzas médio de $1,96 \%(0,68-3,89)$. Comparando-se o carvão comercial de madeira com o carvão produzido com espécies de bambu, pode-se constatar que as espécies Phyllostachys edulis e Phyllostachys nigra produzem carvões com maior densidade relativa aparente média. Os carvões de bambu, com exceção do Phyllostachys edulis, tiveram menor teor de umidade. Somente cinco marcas de carvão comercial tiveram poder calorífico superior maior que os valores registrados para as espécies de bambu, sendo que o carvão de bambu teve menores valores de teor de voláteis e maior quantidade de carbono fixo.

\section{Agradecimentos}

Ao Conselho Nacional de Desenvolvimento Científico e Tecnológico (CNPq) e à Fundação de Amparo à Pesquisa e Inovação do Estado de Santa Catarina (FAPESC) pelo apoio financeiro.

\section{Conclusões}

A qualidade do carvão vegetal variou entre as espécies de bambu, tendo como único ponto negativo o alto teor de cinzas para uso doméstico do carvão vegetal.

Houve alta correlação positiva entre a densidade básica dos colmos e a densidade relativa aparente do carvão vegetal, sendo que as espécies com maior densidade básica produziram carvão mais denso.

A espécie Bambusa vulgaris apresentou o maior rendimento no processo produtivo, porém gerou carvão mais leve e com pior desempenho energético.

Entre as espécies avaliadas, o Phyllostachys nigra foi a que produziu o carvão vegetal com a melhor qualidade energética e com boas propriedades físicas e rendimento gravimétrico.

A qualidade do carvão vegetal varia em relação à altura do colmo. O topo dos colmos, que é o resíduo que pode ser destinado para a produção de carvão em plantios comerciais de bambu para múltiplos usos, apresentou maior rendimento no processo produtivo e carvão com maior densidade. Porém a qualidade energética do carvão foi inferior em relação à base e posição mediana dos colmos. 


\section{Referências}

AMERICAN SOCIETY FOR TESTING AND MATERIAL. ASTM 1762: Standard Test Method for Chemical Analysis of Wood Charcoal. West Conshohocken, 2013. 2 p.

ANDRADE, J. K. B. et al. Productive potential of species Bambusa vulgaris Schrad. charcoal grown in Timon, Ma. Revista Verde, Pombal, v. 10, n. 3, p. 29-33, 2015.

ASSOCIAÇÃO BRASILEIRA DE NORMAS TÉCNICAS. NBR 11941: Madeira - Determinação da densidade básica. Rio de Janeiro, 2003a. 6 p.

ASSOCIAÇÃO BRASILEIRA DE NORMAS TÉCNICAS. NBR 14929: Madeira - Determinação do teor de umidade de cavacos - Método por secagem em estufa. Rio de Janeiro, 2003b. 3 p.

BAZ, R. J. Q. et al. Physico-chemical properties of bamboo charcoals from species Bambusa vulgaris var vittatta, Dendrocalamus asper and Phyllostachys pubescens. Revista Brasileira de Energias Renováveis, Curitiba, v. 8, n. 3, 2019.

BERNDSEN, R. S. et al. Propriedades físicas do bambu-mossô (Phyllostachys pubescens Mazel ex H. de Lehaie) em diferentes idades e posições do colmo. Floresta, Curitiba, v. 40, n. 1, p. 183-192, jan./mar. 2010.

BRAND, M. A. et al. Qualidade do carvão vegetal para o consumo doméstico comercializado na região serrana sul de Santa Catarina. Revista Árvore, Viçosa, MG, v. 39, n. 6, p. 1165-1173, 2015.

BRASIL. Lei no 12.484 8 de setembro de 2011. Disponível em: https://www.planalto.gov.br/ ccivil_03/_ato2011-2014/2011/lei/112484.htm. Acesso: 12 Jun. 2015.

CAMPOS, R. F. et al. Espécies exóticas de bambu no município de São Mateus do Sul, Paraná. Cadernos de Agroecologia, Pelotas, v. 11, n. 2, dez. 2016.

DEUTSCHES INSTITUT FÜR NORMUNG. DIN 51900: determining the gross calorific value of solid and liquid fuels using the bomb calorimeter, and calculation of net calorific value. Berlim, 2000. 7 p.

GUERRA, S. P. S. et al. Mechanized harvesting of bamboo plantations for energy production: Preliminary tests with a cut-and-shred harvester. Energy for Sustainable Development, Portland, v. 34, p. 62-66, 2016.

INTERNATIONAL NETWORK FOR BAMBOO AND RATTAN. Can bamboo transform bioenergy? 15 oct. 2014. Disponível em: http://www.inbar.int/2014/10/can-bamboo-transformbioenergy/. Acesso em: 12 jun. 2015.

LIU, Z.; FEI, B.; JIANG, Z. Combustion characteristics of bamboo-biochars. Bioresource Technology, Lucknow, v. 167, p. 94-99, 2014.

MARAFON, A. C.; AMARAL, A. F. C.; LEMOS, E. E. P. Caracterização de espécies de bambu e outras biomassas com potencial para a geração de energia térmica. Pesquisa Agropecuária Tropical, Goiânia, v. 49, p. e55282-e55282, 2019.

MELO, R. R. de et al. Physical mechanical properties of wood-bamboo particleboard. Ciência Rural, Santa Maria, v. 45, n. 1, p. 35-42, jan. 2015.

NEVES, T. A. et al. Avaliação de clones de Eucalyptus em diferentes locais visando à produção de carvão vegetal. Pesquisa Florestal Brasileira, Colombo, v. 31, n. 68, p. 319, 2011.

OYEDUN, A. O.; GEBREEGZIABHER, T.; HUI, C. W. Mechanism and modelling of bamboo pyrolysis. Fuel processing technology, Western Australia, v. 106, p. 595-604, 2013.

PEREIRA NETO, J. S. et al. Aplicação do bambu nas construções rurais. Revista Educação Agrícola Superior, Campina Grande, v. 24, n. 2, p. 67-77, 2009. 
RAMBO, M. K. D.; SCHMIDT, F. L.; FERREIRA, M. M. C. Analysis of the lignocellulosic components of biomass residues for biorefinery opportunities. Talanta, Seattle, v. 144, p. 696703, 2015.

SANTANA, G. M. et al. Carvão ativado a partir de resíduos de bambu (Bambusa vulgaris) utilizando CO2 como agente ativante para adsorção de azul de metileno e fenol. Ciência Florestal, Santa Maria, v. 29, n. 2, p. 769-778, 2019.

SANTOS, R. C. dos et al. Correlações entre os parâmetros de qualidade da madeira e do carvão vegetal de clones de eucalipto. Scientia Forestalis, Piracicaba, v. 39, n. 90, p. 221-230, 2011.

SÃO PAULO (Estado). Secretaria de Agricultura e Abastecimento de São Paulo. Resolução n 10 SAA, de 11 de julho de 2003. São Paulo, 2003.

TOMBOlATO, A. F. C.; GRECO, T. M.; PINTO, M. M. Dez espécies de bambus exóticos mais comuns no paisagismo no Brasil. Revista Brasileira de Horticultura Ornamental, Campinas, v. 18, n. 2, p. 105-114, 2012.

VALE, A. T.; MOREIRA, A. C. O.; MARTINS, I. S. Avaliação do potencial energético de Bambusa vulgaris em função da idade. Floresta e Ambiente, Rio de Janeiro, v. 24, p. 1-9, 2017. 